
\title{
R Research S Surare \\ Tracking normal fluid flow in He II with unsupervised machine learning
}

\section{Xin Wen}

University of Tennessee at Knoxville https://orcid.org/0000-0001-7892-5095

\section{Landen McDonald}

Oak Ridge National Laboratory

\section{J. Pierce}

Oak Ridge National Laboratory

\section{Wei Guo}

Florida State University https://orcid.org/0000-0002-9466-3213

Michael Fitzsimmons ( $\sim$ fitzsimmonsm@ornl.gov)

Oak Ridge national Laboratory

\section{Article}

Keywords:

Posted Date: January 18th, 2022

DOI: https://doi.org/10.21203/rs.3.rs-1240914/v1

License: (c) (1) This work is licensed under a Creative Commons Attribution 4.0 International License. Read Full License 


\title{
Tracking normal fluid flow in He II with unsupervised machine learning
}

\author{
X. Wen, ${ }^{1,2,3}$ L. McDonald, ${ }^{2}$ J. Pierce, ${ }^{2}$ W. Guo,,${ }^{4,5}$ and M.R. Fitzsimmons ${ }^{1,2,3,{ }^{*}}$
}

${ }^{1}$ Department of Physics and Astronomy, University of Tennessee, Knoxville, TN 37996, USA.

${ }^{2}$ Oak Ridge National Laboratory, Oak Ridge, TN 37830, USA.

${ }^{3}$ Shull Wollan Center-a Joint Institute for Neutron Sciences, Oak Ridge, TN 37830.

${ }^{4}$ Mechanical Engineering Department, FAMU-FSU College of Engineering, Florida State University, Tallahassee, FL 32310, USA.

${ }^{5}$ National High Magnetic Field Laboratory, Tallahassee, FL 32310, USA.

*Corresponding author: M.R. Fitzsimmons mf3@ornl.gov

Abstract - Time dependent observations of point-to-point correlations of the velocity vector field (structure functions) are necessary to fully understand and model fluid flow around complex objects. Using thermal gradients, we induced flow of the normal fluid component of liquid He II and observed the flow by recording fluorescence of $\mathrm{He}_{2}^{*}$ excimers produced by neutron capture throughout a $\sim \mathrm{cm}^{3}$ volume. We applied an unsupervised machine learning algorithm to identify individual excimer clouds and then track their motion with millimeter and millisecond precision using a purpose-built correlation metric. Owing to the fact excimers are produced over a large region, the data are sparse in comparison to other techniques used to produce excimers. Machine learning is crucially important to track flow represented by sparse data-a situation encountered in other fields of science - and its importance will increase as improvements are made to overcome the sparsity of data.

The United States Government retains and the publisher, by accepting the article for publication, acknowledges that the United States Government retains a non-exclusive, paid-up, irrevocable, world-wide license to publish or reproduce the published form of this manuscript, or allow others to do so, for United States Government purposes. 
Previously, we demonstrated the ability to produce copious $H e_{2}^{*}$ excimers in He II using neutron capture, to excite the excimers with lasers and to observe the resulting fluorescence. ${ }^{1}$ The demonstration was performed in a quiescent environment (i.e., one with very little fluid motion) of an enclosed glass bulb. Acquisition of such images taken during flow around objects is expected to provide data to test and validate theoretical ${ }^{2}$ and computer models that describe the development, intensity, and internal structure of turbulent flow beyond simple systems (e.g., flow over a smooth flat plate). ${ }^{3}$ Especially important are data with good spatial resolution of three dimensional (3D) velocity vector fields acquired simultaneously over a field of view with size well in excess of the object using a technique that minimizes perturbations to flow. ${ }^{4,5,6}$ Data to monitor turbulent flow will become increasingly numerous and complex as state-of-the-art camera technologies become more affordable necessitating the need for new visualization and analysis techniques. Here, we apply machine learning to identify and quantify flow. For the case of fluid flow, the technique may facilitate tests of fundamental concepts in turbulence models applied to engineering challenges ${ }^{7,8,9}$ and enable research in quantum turbulence related to astrophysics ${ }^{10}$ and cosmology. ${ }^{11}$ More broadly, the technique to use machine learning to identify clusters of objects and the correlation metric developed here to infer movement of the clusters may be applicable to studies beyond fluid flow, e.g., migration of herds of animals ${ }^{12}$, traffic flow ${ }^{13}$ and spread of transmissible diseases ${ }^{14}$.

We show the ability to control the flow of the normal component of He II and to track the flow across a 1 $\mathrm{cm}$ by $1 \mathrm{~cm}$ field of view. Motion is evident in the images of the fluorescence and through tracking of individual excimer clouds identified using an unsupervised machine learning clustering algorithm. We measured the correlation between the velocity flow field and power applied to heaters that create thermal gradients. Evidence for flow is unequivocal. We demonstrate flow brings excimers from a portion of the He cryostat illuminated by the neutron beam into the region of the cryostat that is in the shadow of the neutron beam - a region in which excimers cannot be produced. All other approaches using $H e_{2}^{*}$ excimers to track flow, monitor the evolution of fluorescence as $\mathrm{He}_{2}^{*}$ excimers move from an initially confined region. ${ }^{15,16}$ Our approach produces $H e_{2}^{*}$ excimers throughout a large volume, then we identify clusters of $H e_{2}^{*}$ excimers with 
machine learning and then track the clusters. The new approach lays the foundation to observe flow that fully encompasses $\mathrm{cm}$-sized objects and provides opportunities to observe point-to-point correlation in space and time of the velocity vector field (dynamic structure functions).

A detailed description of the experimental setup is given in Ref. [1]. Briefly, a cryostat with windows transparent to infrared light contains a glass bulb that itself contains a mixture of ${ }^{3} \mathrm{He}$ and ${ }^{4} \mathrm{He}$ in the ratio of 1 to 1818 (pressure at $300 \mathrm{~K}$ is $67 \mathrm{kPa}$ ). The bulb was cooled by a liquid He bath to $1.6 \mathrm{~K}$. Neutron capture by one ${ }^{3} \mathrm{He}$ atom yields ionizing radiation in the form of a proton and triton with energies of 573 and $191 \mathrm{keV}$, respectively, and mean free paths in liquid He of 59 and $15 \mu \mathrm{m}$, respectively. ${ }^{17}$

Protons and tritons create helium ions $\mathrm{He}^{+}$, electrons and excited state $\mathrm{He}^{*}$ atoms. One $\mathrm{He}^{+}$and 0.45 $H e^{*}$ are produced ${ }^{18}$ for every $43(1) \mathrm{eV}$ deposited by $\alpha$ and $\beta$ particles, ${ }^{19,20}$ which we assume is the case for tritons and protons. After thermalization, ion-electron pairs in proximity to ground state $\mathrm{He}$ atoms can form $\mathrm{He}$ dimers: $e^{-}+\mathrm{He}^{+}+\mathrm{He} \rightarrow e^{-}+\mathrm{He}_{2}^{+}$and then combine to form helium excimer molecules: $e^{-}+\mathrm{He}_{2}^{+} \rightarrow$ $H e_{2}^{*} \cdot{ }^{21,22,23}$ In the absence of spin correlation between the electron and dimer, which is reasonable for very dense ionization events produced by heavy nuclei, $75 \%$ of the $H e_{2}^{*}$ should be in the spin triplet state, $a_{3} \Sigma_{u}$, and the remainder in the spin single state. $H e^{*}$ atoms can also create helium excimer molecules: $\mathrm{He}^{*}+\mathrm{He} \rightarrow$ $H e_{2}^{*}{ }^{24}$ Sato et al. ${ }^{18}$ calculate $17 \%$ of the $H e^{*}$ occupy the spin triplet state. Because a spin-flip transition is forbidden, $17 \%$ of the $H e_{2}^{*}$ created from $H e^{*}$ in proximity to ground state atoms will be in the spin triplet state. Thus, we expect for every neutron captured not more than $\frac{764 \mathrm{keV}}{43 \mathrm{eV}}(1 \times 0.75+0.45 \times 0.17) \sim 14,700 \mathrm{He} e_{2}^{*}$ excimers will be produced in the $a_{3} \Sigma_{u}$ spin triplet state. On account that so many excimers are created in a small region ( $764 \mathrm{keV}$ deposited along $74 \mu \mathrm{m}$, i.e., $\sim 10 \mathrm{keV} / \mu \mathrm{m}$ from neutron capture compared to $40 \mathrm{eV} / \mu \mathrm{m}$ for a $500 \mathrm{keV}$ electron ${ }^{25,26}$ ), some excimers will be lost due to Penning ionization. ${ }^{27}$

The $a_{3} \Sigma_{u}$ spin triplet state can be excited with a pair of $905 \mathrm{~nm}$ photons to emit a $640 \mathrm{~nm}$ photon (the fluorescence), which can be recorded by cameras. ${ }^{28,29}$ Because the normal component of He II is viscous, this component drags the excimers with it; ${ }^{30}$ thus, the location of the fluorescence as a function of time provides a means to record the motion of the normal component. The bulb has a footprint of $1 \mathrm{~cm}$ by $1 \mathrm{~cm}$ and is $3.6 \mathrm{~cm}$ 
tall (Fig. S1 [31]). Only $1 \mathrm{~cm}$ of the bulb's length near its bottom is visible to the camera. While excimers are produced by the neutron beam throughout the bottom $1 \mathrm{~cm}^{3}$ of the bulb, only excimers within $1 \mathrm{~mm}$ of the plane that bisects the bulb and is perpendicular to the camera's axis are illuminated by the lasers and can fluoresce (Fig. S1 [31] ). This plane is also the focal plane of the camera.

The experimental setup for the present experiment differs from Ref. [1] in two ways. First, a 6.4-mm thick plate of boron-nitride $(\mathrm{BN})$ (an effective neutron absorber) was placed on the front surface of the cryostat to block the neutron beam from entering either the top or alternately the bottom half of the glass bulb, thus, forming a neutron shadow (Fig. S1 [31]). There are no excimers created in the shadow of the neutron beam. Thus, fluorescence observed in the shadowed region must originate from excimers created in the unshadowed region that moved into the shadowed region. Second, a fiberglass (G-10) rod was inserted into the bulb but displaced away from the laser sheet by about $1 \mathrm{~mm}$. The rod was wrapped with $40 \mathrm{~cm}$ of 32 AWG Nichrome wire as two separate coils $1 \mathrm{~cm}$ above and below the center of the camera's field of view (Fig. S1 [31]). The coils could be energized independently to produce a thermal gradient. By passing current through a coil, heat was created and transported away from the coil via thermal counterflow: the normal fluid moves at a speed proportional to the heat applied, and the superfluid moves in the opposite direction to ensure no net mass flow. ${ }^{32}$ We measured the fluorescence of the excimers for power ranging from 0 to $40 \mathrm{~mW}$ applied either to the top or bottom heater, with the $\mathrm{BN}$ absorber either blocking the neutron beam from entering either the top or bottom of the bulb, i.e., four combinations. Regardless of the position of the BN absorber, if the neutron beam hit the portion of the bulb with the heater turned on, then fluorescence was observed to increase with time in the shadow of the neutron beam. A movie of the fluorescence recorded by the camera's field of view as a function of time for power of $10 \mathrm{~mW}$ applied to the bottom heater shows the induced flow (Fig. S2 [31]).

The fluorescence integrated over the horizontal dimension of the camera (i.e., of each image in the movie Fig. S2 [31]) is plotted as a function of vertical position and time in (left) Fig. 1. The region of the bulb in the neutron shadow corresponds to vertical position $>5 \mathrm{~mm}$ (denoted by the transparent shadow effect in Fig. 1) relative to the center of the field of view at $0 \mathrm{~mm}$. The neutron beam was turned on at time $=5 \mathrm{~s}$, and the 
recording started at time $=8 \mathrm{~s}$. Once the neutron beam was turned on, excimers begin to form in the unshadowed region (laser light enters the cryostat from the side opposite to the BN neutron beam blocker, so only the neutron beam is blocked, see Fig. S1 [31]). The lasers (needed to excite the excimers) were turned on at $t_{l}=10 \mathrm{~s}$, and fluorescence was immediately observed. Note the absence of fluorescence for vertical position $>5 \mathrm{~mm}$ for times up to $\sim 12 \mathrm{~s}$. The heater was turned on at $t_{h}=12 \mathrm{~s}$. Shortly thereafter, fluorescence was observed in the neutron shadow, and the fluorescence moved to larger vertical positions (into the shadow of the neutron beam) with increasing time. Eventually, the field of view became saturated with fluorescence (right, Fig. 1).

The red line in Fig. 1 is the slope of the line that best fits the peak of the intensity profile vs. vertical position for constant time. The inverse of the slope is the component of the velocity of the normal fluid flow along the vertical direction (in this instance motion to positive vertical position "up" in Fig. S2 [31]). Shown in Fig. 2 is the normal fluid (vertical) velocity as a function of heater power. The error bars represent the standard deviation of the velocities obtained from repeated measurements. Landau and Lifshitz ${ }^{33,34}$ provide an equation $q=\rho S T V$ that relates the heat flux, $q$, to normal fluid velocity, $V$, through the mass density of liquid He, $\rho$, its entropy, $S$, and temperature $T$. We estimated a value of $q$ as the ratio of the heater power, $P$, to the surface area of the coil of $A=2.4 \mathrm{~cm}^{2}$. Using values of $\rho=1.452 \mathrm{~g} / \mathrm{cm}^{3}, S=0.2824 \mathrm{~J} / \mathrm{g} \cdot \mathrm{K}$ for $\mathrm{T}=1.6 \mathrm{~K},{ }^{35}$ we calculated the expected value of $V$ vs. power $\left(V=\frac{1}{A \rho S T} P\right)$, which is shown as the green line in Fig. 2 . The blue line is the error-weighted best fit to the data subject to the constraint $V=0$ for $P=0$. Because not all windings of the coil were fully exposed, the effective surface area of the coil in the experiment is less than our estimate of $A$, consequently, the slope of the fitted line (blue) is somewhat larger than $V(P)$ (green line). Assuming a length scale, $L$, comparable to the field of view $(1 \mathrm{~cm})$ and using the kinematic viscosity of liquid $\mathrm{He}$ at $1.6 \mathrm{~K}$ of $v=$ $9 \times 10^{-9} \mathrm{~m}^{2} / \mathrm{s}$ [35] and the maximum observed velocity $\sim 3 \mathrm{~mm} / \mathrm{s}$ (Fig. 2), the maximum Reynolds number achieved in this experiment is $R e=\frac{V L}{v} \sim 3333$. This value is greater than $R e \sim 2000$ needed to observe turbulent flow in a pipe geometry. ${ }^{36}$ 
Electrons produced by electric discharge from needles ${ }^{29}$ or laser induced ionization ${ }^{37}$ are means to initially produce high densities (e.g., $1.4 \times 10^{9} \mathrm{~cm}^{-3}$ [29]) of excimers in localized regions (e.g., $\sim 0.005 \mathrm{~cm}^{3}$, see Fig. 2 of Ref. [38]), although Penning ionization of the excimers is expected to reduce the density with time. The images of fluorescence in Ref. [38] are easily seen, i.e., well-defined, and typical of all other approaches to track flow using $H e_{2}^{*}$ excimers. In contrast, the images Fig. S2 [31] are not well-defined. The lack of definition is because neutron capture can occur anywhere in the $1 \mathrm{~cm}^{3}$ volume of the bulb, and the neutron capture rate is low, consequently, the data are sparse. The neutron capture rate, $R=22(4) \mathrm{n} / \mathrm{s} \cdot \mathrm{cm}^{3}$, is the product of the neutron beam intensity, $1.3(2) \times 10^{5} \mathrm{n} / \mathrm{s} \cdot \mathrm{cm}^{2}$ integrated over neutron wavelengths from 3.07 to $5.53 \AA$ used in the experiment, the average neutron cross-section of ${ }^{3} \mathrm{He} \bar{\sigma}=13511$ barns for this wavelength range, and the density of ${ }^{3} \mathrm{He}, 1.26(8) \times 10^{16} \mathrm{~cm}^{-3}$. Using the upper limit of 14,700 (spin triplet state) excimers per neutron capture, and $R$, excimers are produced at a rate of $\sim 3 \times 10^{5} \mathrm{~s}^{-1} \mathrm{~cm}^{-3}$. This value corresponds to a density averaged over the volume of liquid illuminated by the neutron beam. In proximity to the capture event, the density will be much higher, and excimer production will be diminished by excimer loss processes, e.g., Penning ionization, recombination, etc. What the neutron capture approach lacks in terms of (localized) signal compared to other approaches is possibly offset by the opportunity to monitor flow simultaneously about large scale structures in three dimensions. ${ }^{39}$ To achieve this goal a means is needed (1) to identify individual excimer clusters in an image and (2) to observe the motion of the clusters from one image to the next. To identify excimer clusters, we applied a machine learning algorithm to each image (e.g., those shown in Fig. S2 [31]). To track the clusters, we developed a metric to estimate the likelihood that a cluster in one image is correlated (or the same) as a cluster in the subsequent image (i.e., the image taken $1 /(55.6 \mathrm{~Hz})$ later).

To facilitate automation, an unsupervised clustering algorithm is desirable. Further, to avoid biasing the number of clusters in any given image (because the intensity of the fluorescence can change with time, see (right) Fig. 1), an algorithm that does not constrain a priori the number of clusters in an image is preferred. We investigated many algorithms and found the Mean Shift clustering algorithm ${ }^{40}$ worked best. This approach identifies arbitrarily shaped objects as clusters and obtains the centroids of the clusters using a well-established 
pattern recognition procedure - the Mean Shift procedure. ${ }^{41}$ The approach regards the image as a probability density function of a parameter-in our case the positions of fluorescence in the same frame, i.e., at fixed time. The approach differs from another popular class of clustering approaches that use hierarchical clustering based on proximity measures. ${ }^{42}$

An example of the application of the algorithm to data collected for time slice of $17.18 \mathrm{~s}$ of Fig. 1 is shown in (left) Fig. 3. The large symbols represent the centroid obtained for the cluster; the small dots of the same color show excimers identified for the colored cluster. A movie of the clusters vs. time showing the upward motion of the clusters is presented in Fig. S3 [31]. Fig. S3 [31] uses the same information shown in Fig. S2 [31]. The average number of clusters identified per frame from Fig. S2 [31] was 6(2). The average number of excimer events in each cluster was 9(7). The maximum signal in Fig. 1(right) corresponds to $\sim 80$ excimers per frame, while the signal recorded in the portion of the cryostat illuminated by the neutron beam (thus producing excimers) corresponds to $\sim 32$ excimers per frame. Previously, we argued the upper limit to the number of excimers produced by capture of one neutron was 14,700 . The number of neutrons captured in the laser sheet is 2 per second. During the first second after the neutron and laser beams are turned on, we expect not more than 30k excimers to fluoresce. Assuming all fluoresce, the fluorescence captured by the camera will correspond to an upper limit equal to the product of 30k excimers, the ratio of the solid angle of the lens to $4 \pi$ $\left(=\frac{\pi 1.5^{2}}{7^{2}} / 4 \pi\right)$, and the quantum efficiency of the image intensifier $(0.36[43])$. The product—an upper limit—is 124 excimers. The value is greater than the number of excimers observed at $t_{l}$ of $\sim 32$ for Figs. 1 and S2 [31]. We note Penning ionization will reduce observed value, and $t_{l}$ was preceded by 5 seconds worth of neutron beam exposure before the laser were turned, which should increase the observed value. Nevertheless, the calculated and observed values are reasonably consistent.

Next, we compare centroids of the $i$-th image taken at time $t_{i}$ with those in the next image at $t_{i+1}$. The comparison involved summing over the distances between centroids in the first image with those in the second image for every combination of cluster pairs (a pair is a cluster in the first frame and a second cluster in the next frame) that did not exceed a distance of $D$ apart. Given the size of a cluster and the speed of the flow, there are 
values of $D$ that are not reasonable. The influence of choice of $D$ on the metric is shown in S4 [31]. There are potentially many combinations that fulfill this metric. The combination yielding the smallest sum of $D$ was chosen as the solution that identified the same cluster(s) in adjacent frames. Application of this metric, called the correlation metric, while simple, can yield erroneous results. For example, the production of a new excimer cloud by neutron capture in one frame could be mistaken for motion of an excimer cloud from the previous frame. However, this situation cannot be realized for clouds that are tracked in the shadow of the neutron beam. The movement of the centroid of the cluster from one frame to the next is the velocity in two dimensions of the cluster. Using the correlation metric and a value of $D=234 \mu m(6 \times$ the camera pixel dimension $)$, two cluster pairs (right) Fig. 3 were identified for images taken at 17.17 and $17.18 \mathrm{~s}$ (the latter is shown in (left) Fig. 3). In (right) Fig. 3 an arrow shows the motion of the centroid of one cluster in the first frame to the next. Centroids of clusters that are not connected by an arrow are separated by a distance greater than $D$, thus, such clusters are not considered to be the same cluster that moved from one frame to the next.

Once the same cluster was identified in sequential images, the displacements in the vertical and horizontal directions (within the camera's focal plane), i.e., the velocity vector of the cluster times the period corresponding to the camera's frame rate were calculated. The results are shown in (left) Fig. 4 and (left, Fig. S5 [31]) for the vertical and horizontal directions, respectively. On the right side of these figures are histograms of the displacements for times integrated from (red) 12 to $15 \mathrm{~s}$ and (blue) 12 to $19 \mathrm{~s}$. For times during which flow was observed in Fig. 1 (from 12 to 19s), the histogram is skewed towards positive (upward) displacement (consistent with Figs. 1 and S2 [31]). Motion of the excimer clusters identified with machine learning and the correlation metric applied to data shown in Fig. S2 [31] is shown as a movie in Fig. S6 [31]. The consistency between motion of the individual clusters (Fig. S6 [31]) and motion of the fluorescence (Fig. S2 [31]) demonstrate the opportunity of the approach to quantify the velocity vector field flow in two dimensions extracted from individual excimer clouds and sparse data.

In summary, we have demonstrated the ability to observe the motion of $H e_{2}^{*}$ excimers through their fluorescence across a $1 \mathrm{~cm}^{2}$ field of view. We applied an unsupervised machine learning algorithm and 
correlation metric to identify and track the centroids of individual excimer clusters in the focal plane of the camera as a function of time. This allows us to obtain the velocity vector field of the flow from sparse data with a spatial resolution of $\sim 39 \mu \mathrm{m}$, a temporal resolution of $18 \mathrm{~ms}$ across $1 \mathrm{~cm}$ in two dimensions. With additional lasers and cameras, the approach can be extended to observe flow in three dimensions. The density of excimer clusters can be increased by a factor of $10 \mathrm{x}$ by increasing the concentration of ${ }^{3} \mathrm{He}$ accordingly. The increased density of ${ }^{3} \mathrm{He}$ would be $\sim 1 \%$ of the roton density and thus not expected to affect the hydrodynamics of He II at 1.7 K. ${ }^{1}$ With more excimer clusters, the spatial resolution will be improved. However, with more excimer clusters to track, the correlation metric may provide ambiguous results (cluster confusion). Cameras for neutron imaging with Timepix3 [44] integrated chips record scintillation events with spatial resolution of 10's of microns in event mode - meaning the absolute times of events are recorded continuously (with $1.6 \mathrm{~ns}$ precision). Use of event mode cameras would improve the reliability of the machine learning algorithm and the correlation metric for fluorescent imaging applications of $\mathrm{He}_{2}^{*}$. For example, with continuous recording of event times, the value of $D$ could be reduced increasing the confidence of the correlation metric — essentially individual excimer clusters identified with machine learning could be continuously tracked or alternatively, time could be included in the machine learning algorithm as an additional dimension. A further advantage of continuous operation is that the repetition rate of the images is constrained by the frequency of the $905 \mathrm{~nm}$ laser, $1 \mathrm{kHz}$, and not the bandwidth of the camera. The small bandwidth of the camera used presently (e.g., to record the images of Fig. S2 [31]), only allowed data to be recorded from three of the 18 laser pulses (to form one image), while discarding the remaining 15 laser pulses. Faster cameras enable images from every laser pulse to be recorded - a 6-fold increase of data acquisition.

\section{Acknowledgments}

This research used resources at the Spallation Neutron Source, a DOE Office of Science User Facility operated by the Oak Ridge National Laboratory. ORNL is managed by UT-Battelle, LLC, for the U.S. Department of Energy under Contract No. DE-AC05-00OR22725. Valuable discussions with J. Hodges and M. Doucet (ORNL) are gratefully acknowledged. X.W. acknowledges support from the Shull Wollan Center 
Graduate Research Fellowship program and Graduate Advancement, Training and Education (GATE) program of University of Tennessee. W.G. acknowledges the support from the National Science Foundation under Grant No. DMR-2100790 and the National High Magnetic Field Laboratory, which is supported by National Science Foundation Cooperative Agreement No. DMR-1644779 and the state of Florida. 


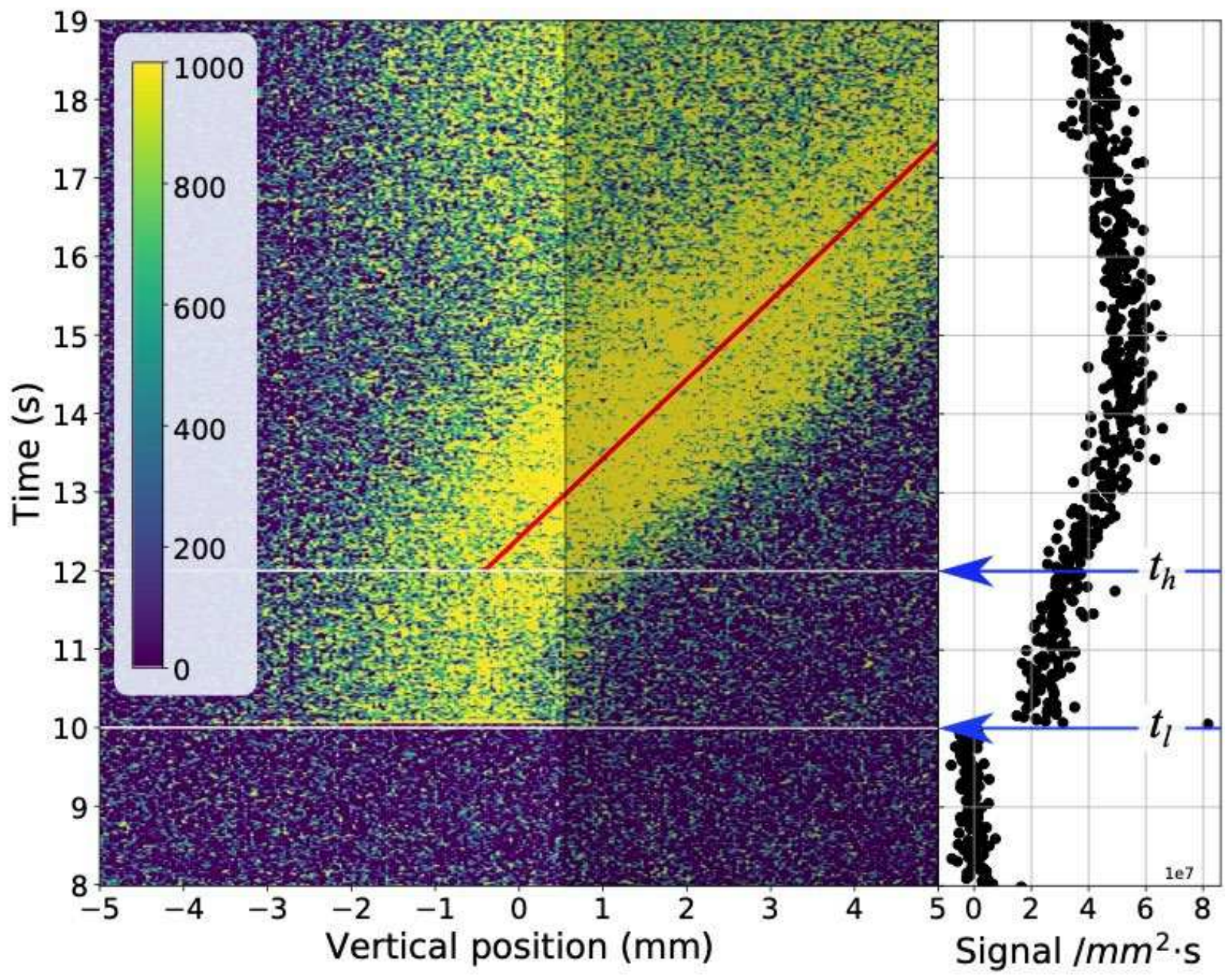

Fig. 1 (left) Excimer fluorescence integrated over the horizontal field of view per second recorded versus time and vertical position. Only the region for position $<0.5 \mathrm{~mm}$ is illuminated by the neutron beam. The red line the best fit to the peak of the fluorescence for constant time. The inverse of the slope of the red line is the velocity of the flow for a heater power of $10 \mathrm{~mW}$ (this case). (right) Fluorescence integrated over the entire field of view vs. time. The neutron shutter was opened at $5 \mathrm{~s}$, the laser shutter opened at $t_{l}=10 \mathrm{~s}$, and the bottom heater energized at $t_{h}=12 \mathrm{~s}$. Flow is evident as movement of the fluorescence from the region in which excimers are produced to one in which no excimers are produced (in the neutron shadow). 


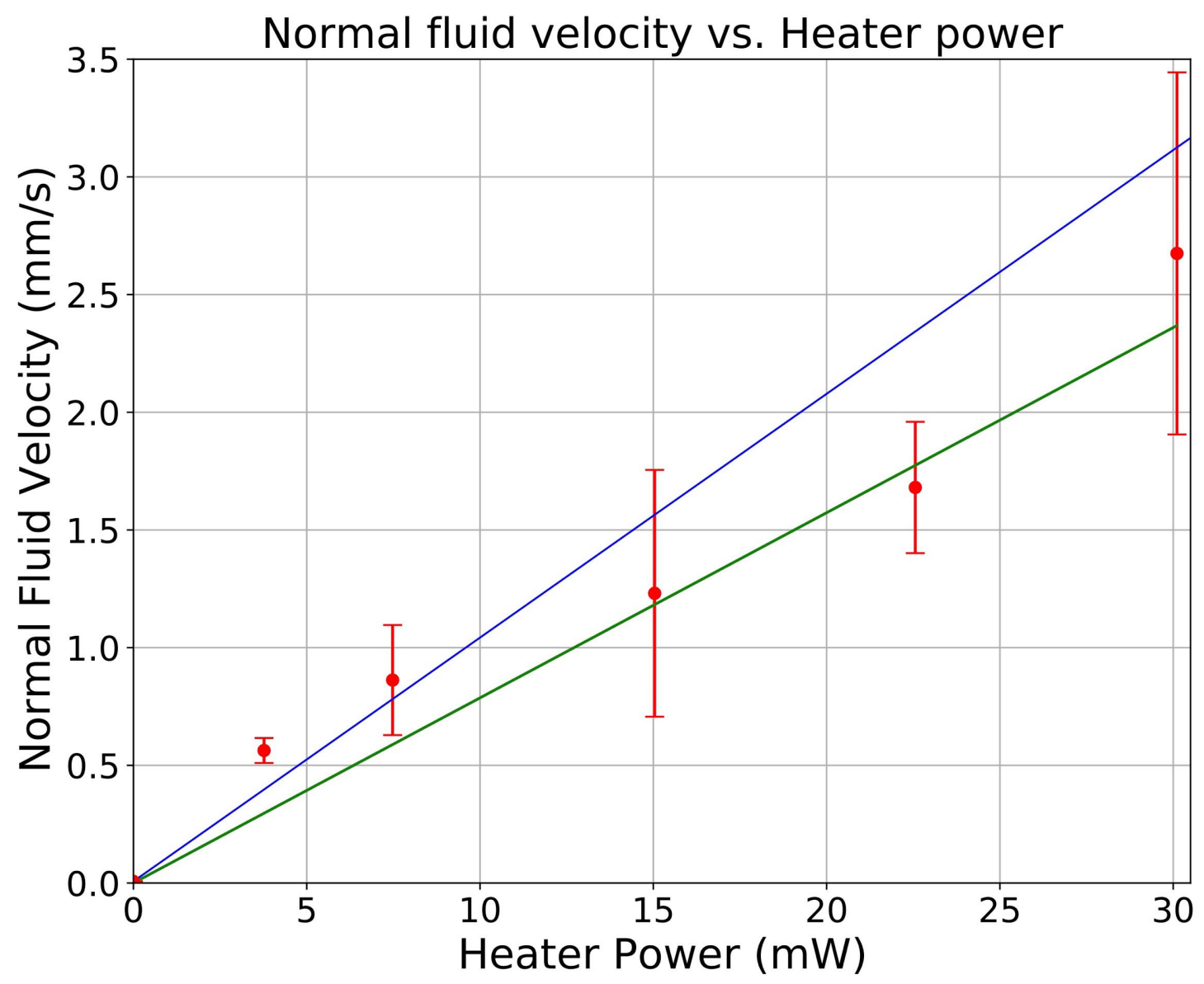

Fig. 2 Normal fluid velocity obtained from the flow rate of fluorescence vs. heater power. The green line was obtained using Landau and Lifshitz's equation for heat flux in $\mathrm{He} \mathrm{II},{ }^{33,30}$ and the blue line is the best weighted fit to the data. The error bars represent the standard deviation of measurements of the same power. 

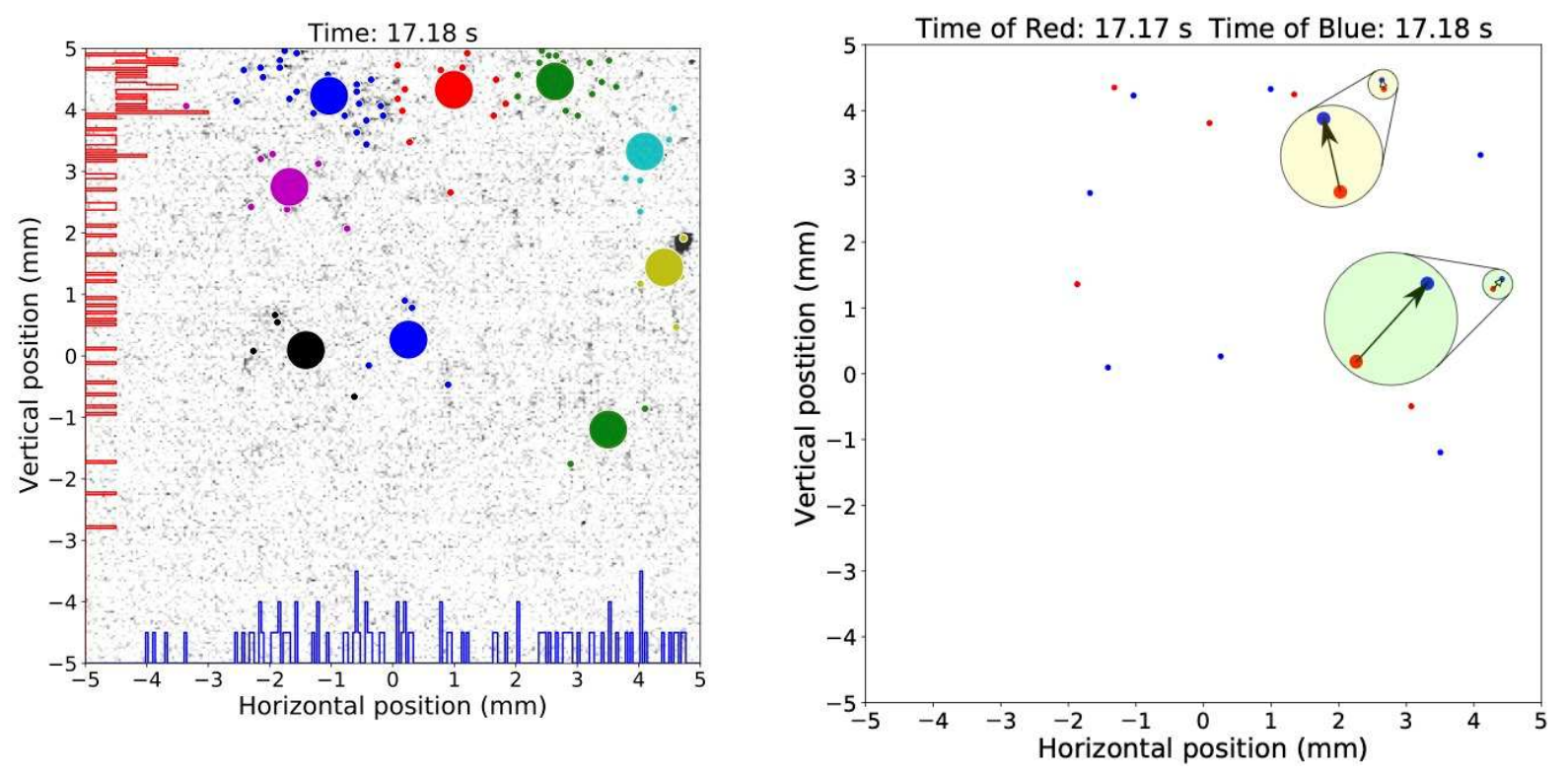

Fig 3 (left) Excimer fluorescence color-coded (small dots) using the MeanShift clustering algorithm (bottom heater power $=10 \mathrm{~mW}$ ). The large dots represent the position of the cluster centroid. (right) Positions of centroids for times of 17.17 and $17.18 \mathrm{~s}$ corresponding to red and blue dots, respectively. In this example, two pairs of red and blue centroids were identified as being the same clusters that moved during the time 17.17 to $17.18 \mathrm{~s}$ (shown by the arrows). 


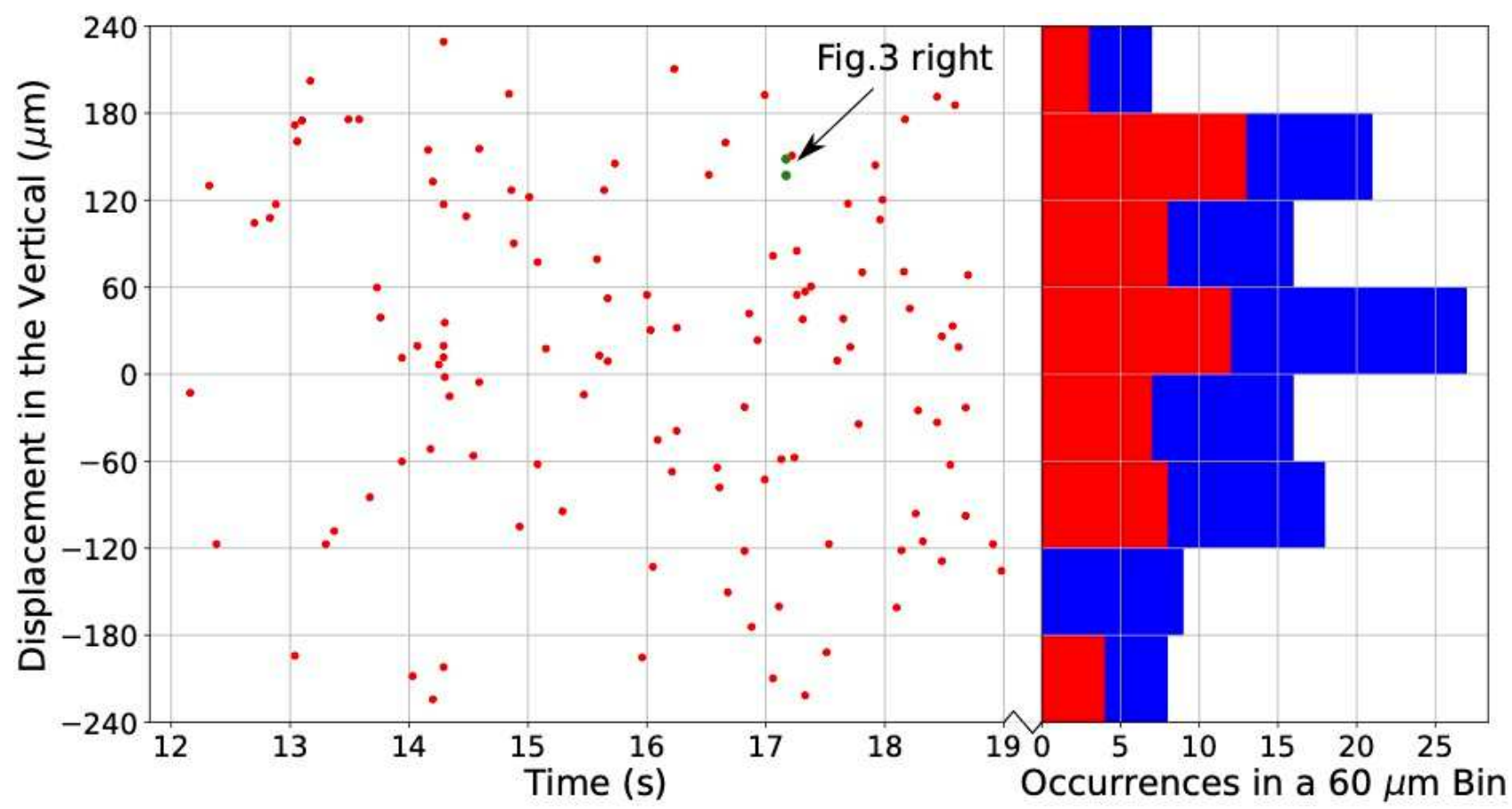

Fig. 4 (left) Vertical displacements (horizontal in Fig. S5) [31] of clusters (within one period between consecutive exposures $=\frac{1}{55.6} \mathrm{~s}$ ) vs. time for conditions with the bottom heater power $=10$ $\mathrm{mW}$. (right) Histograms of the vertical displacements for times of (red) 12 to $15 \mathrm{~s}$ and (blue) 12 to $19 \mathrm{~s}$. The red histogram is skewed to positive displacement—consistent with the image in Fig. 1 and movie Fig S2. The two green dots (see arrows) at $17.17 \mathrm{~s}$ correspond to the vertical displacements shown in Fig. 3 (right). 


\section{References}

${ }^{1}$ X. Wen, Shiran Bao, L. McDonald, J. Pierce, G.L. Greene, Lowell Crow, Xin (Tony) Tong, A. Mezzacappa, R. Glasby, W. Guo and M.R. Fitzsimmons, Phys. Rev. Lett., 124, 134502 (2020).

${ }^{2}$ I. Eames and J.B. Flor, Phil. Trans. R. Soc. A, 369, 702 (2011).

${ }^{3}$ P. Lammers, K. N. Beronov, R.Volkert, G.Brenner and F. Durst, Computers \& Fluids, 35, 1137 (2006).

${ }^{4}$ P. K. Yeung, K. R. Sreenivasan, and S. B. Pope, Phys. Rev. Fluids, 3, 064603 (2018).

${ }^{5}$ T. A. Oliver, N. Malaya, R. Ulerich, and R. D. Moser, Physics of Fluids, 26, 035101 (2014).

${ }^{6}$ T. Zhang and S.W. Van Sciver, Nat. Phys., 1, 36 (2005).

${ }^{7}$ F.G. Schmitt, Comples Rendus Mécanique, 335, 617 (2007).

${ }^{8}$ L. Prandtl, Z Angew. Math Mech, 5, 136 (1925).

9 J. Smagorinsky, Monthly Weather Review, 91, 99-164 (1963).

${ }^{10}$ D. Page, M. Prakash, J. M. Lattimer, and A. W. Steiner, Phys. Rev. Lett., 106, 081101 (2011).

${ }^{11}$ C. Bäuerle, Yu. M. Bunkov, S. N. Fisher, H. Godfrin and G. R. Pickett, Nature, 382, 332-334 (1996).

12 D.A. Wijeyakulasuriya, E.W. Eisenhauer, B.A. Shaby, E.M. PLOS ONE 15(7): e0235750. https://doi.org/10.1371/journal.pone.0235750 (2020)

${ }^{13}$ O. Mohammed and J. Kianfar, 2018 IEEE International Smart Cities Conference (ISC2), pp. 17, https://doi.org/doi: 10.1109/ISC2.2018.8656924 (2018).

${ }^{14}$ Alfred, R., \& Obit, J. H. Heliyon, 7(6), e07371. https://doi.org/10.1016/j.heliyon.2021.e07371 (2021).

${ }^{15}$ W. Guo, M. La Mantia, D.P. Lathrop and S.W. Van Sciver, Proc. of the Nat. Acad. Of Science, 111, 4653 (2014).

${ }^{16}$ M.E. Hayden et al., Phys. Rev. Lett. 93, 105302 (2004). 
${ }^{17}$ C.F. Williamson, J.P. Boujot, J. Picard, Tables of range and stopping power of chemical elements for charged particles of energy 0.05 to $500 \mathrm{MeV}$. Rapport Centre D'Etudes Nucleaires de Saclay, CEA-R3042 pp. 3-151 (1966).

${ }^{18}$ S. Sato, K. Okazaki and Shi-ichi Ohno, Bull. Of the Chem. Soc. Of Japan, 47, 2174 (1974).

${ }^{19}$ N. Ishida, J. Kikuchi and T. Doke, Jpn. J. Appl. Phys., 31, 1465 (1992).

${ }^{20}$ W.P. Jesse and J. Sadauskis, Phys. Rev. 97, 1668 (1955).

${ }^{21}$ N. D’Angelo, Phys. Rev. 121, 505 (1961).

${ }^{22}$ A.B. Callear and R.E.M. Hedges, Nature 215, 1267 (1967).

${ }^{23}$ C.M. Surko and F. Reif, Phys. Rev. 175, 229 (1968).

${ }^{24}$ W. Guo and D.N. McKinsey, Phys. Rev. D, 87, 115001 (2013).

${ }^{25}$ M. J. Berger, J. S. Coursey, M. A. Zucker, and J. Chang, ESTAR, PSTAR, and ASTAR:

Computer Programfor Calculating Stopping-Power and Range Tables for Electrons, Protons, and Helium Ions (National Institute of Standards and Technology, Gaithersburg, 2005) [http://physics.nist.gov/Star].

${ }^{26}$ T.M. Ito, S.M. Clayton, J. Ramsey, M. Karcz, C.-Y. Liu, J.C. Long, T.G. Reddy and G.M. Seidel, Phys. Rev. A 85, 042718 (2012).

${ }^{27}$ D.N. McKinsey, C.R. Brome, J.S. Butterworth, S.N. Dzhosyuk, P.R. Huffman, C.E.H. Mattoni and J.M. Doyle, Phys. Rev. A, 59, 200 (1999).

${ }^{28}$ A. Marakov, J. Gao, W. Guo, S.W. Van Sciver, G. G. Ihas, D. N. McKinsey, and W. F. Vinen, Phys. Rev. B 91, 094503 (2015).

${ }^{29}$ W. Guo, J.D. Wright, S.B. Cahn, J.A. Nikkel and D.N. McKinsey, J. Low Temp. Phys., 158, 346 (2010) https://doi.org/10.1007/s10909-009-0021-x

${ }^{30}$ W.F. Vinen, in Low Temperature Physics, AIP Conf. Proc. 850 (AIP, New York, 2006) p.169.

${ }^{31}$ See Supplemental Material.

${ }^{32}$ W.F.Vinen, The Physics of Superfluid Helium, https://cds.cern.ch/record/808382/files/p363.pdf 
${ }^{33}$ L. D. Landau, The theory of superfluidity of helium II, Journal of Physics USSR, 5, 71, (1941).

${ }^{34}$ L. D. Landau and E. M. Lifshitz, Fluid Mechanics (Pergamon Press, Oxford, 1987).

${ }^{35}$ Russell J. Donnelly, and Carlo F. Barenghi, Journal of Physical and Chemical Reference Data 27, 1217 (1998).

${ }^{36}$ L.F. Moody and N.J. Princeton, Trns. Of the A.S.M.E., 66, 671 (1944).

${ }^{37}$ A.V. Benderskii, R. Zadoyan, N. Schwentner and V.A. Apkarian, J. of Chem. Phys., 110, 1542 (1999).

${ }^{38}$ W. Guo, S.B. Cahn, J.A. Nikkel, W.F. Vinen and D.N. McKinsey, Phys. Rev. Lett., 105, $045301(2010)$.

${ }^{39}$ A similar dichotomy is faced in the choice between using a nuclear reactor-a neutron source with high time averaged flux ideally suited to examine discrete regions of reciprocal space—or a pulsed neutron source - a source with high instantaneous brightness best suited to acquire data from an expansive and continuous range of reciprocal space- for neutron scattering.

${ }^{40}$ D. Comaniciu and P. Meer, IEEE Trans. Pattern Analysis and Machine Intelligence, 24(5), 603 (2002).

${ }^{41}$ K. Fukunaga and L.D. Hostetler, IEEE Trans. Information Theory, 21, 32 (1975).

${ }^{42}$ A.K. Jain, R.P.W. Duin and J. Mao, IEEE Trans. Pattern Analysis and Machine Intelligence, 22(1) 4 (2000).

${ }^{43}$ http://www.pi-j.jp/pdf/manual/PI-MAX3_SystemManual.pdf

${ }^{44}$ https://kt.cern/technologies/timepix3 


\section{Supplementary Files}

This is a list of supplementary files associated with this preprint. Click to download.

- SupplementalMaterial.pdf

- Figures2.mp4

- FigureS3.mp4

- Figures6.mp4 